\title{
Cardiovascular Risk Prediction in Ankylosing Spondylitis: From Traditional Scores to Machine Learning Assessment
}

\author{
Luca Navarini · Francesco Caso · Luisa Costa · Damiano Currado $\cdot$ Liliana Stola $\cdot$ Fabio Perrotta \\ Lorenzo Delfino - Michela Sperti - Marco A. Deriu • Piero Ruscitti • Viktoriya Pavlych • Addolorata Corrado • \\ Giacomo Di Benedetto · Marco Tasso · Massimo Ciccozzi · Alice Laudisio · Claudio Lunardi · Francesco Paolo Can- \\ tatore $\cdot$ Ennio Lubrano · Roberto Giacomelli · Raffaele Scarpa · Antonella Afeltra
}

Received: July 23, 2020 / Accepted: August 28, 2020 / Published online: September 16, 2020

(C) The Author(s) 2020

\section{ABSTRACT}

Introduction: The performance of seven cardiovascular (CV) risk algorithms is evaluated in a multicentric cohort of ankylosing spondylitis (AS) patients. Performance and calibration of traditional CV predictors have been compared with the novel paradigm of machine learning (ML).

Methods: A retrospective analysis of prospectively collected data from an AS cohort has been

Luca Navarini and Francesco Caso contributed equally to this paper.

L. Navarini $(\varangle) \cdot$ D. Currado $\cdot$ L. Stola · A. Afeltra Unit of Allergology, Immunology, Rheumatology, Department of Medicine, Università Campus BioMedico di Roma, Rome, Italy

e-mail: 1.navarini@unicampus.it

F. Caso $(\varangle) \cdot$ L. Costa $\cdot$ M. Tasso $\cdot$ R. Giacomelli Rheumatology Unit, Department of Clinical Medicine and Surgery, School of Medicine, University Federico II of Naples, Naples, Italy e-mail: francescocaso1@yahoo.it; francesco.caso@unina.it

F. Perrotta $\cdot$ E. Lubrano

Academic Rheumatology Unit, Dipartimento di Medicina e Scienze della Salute "Vincenzo Tiberio", Università degli Studi del Molise, Campobasso, Italy

L. Delfino · C. Lunardi

Department of Medicine, University of Verona, Verona, Italy performed. The primary outcome was the first $\mathrm{CV}$ event. The discriminatory ability of the algorithms was evaluated using the area under the receiver operating characteristic (ROC) curve (AUC), which is like the concordancestatistic (c-statistic). Three ML techniques were considered to calculate the $\mathrm{CV}$ risk: support vector machine (SVM), random forest (RF), and k-nearest neighbor (KNN).

Results: Of 133 AS patients enrolled, 18 had a CV event. c-statistic scores of 0.71, 0.61, 0.66, $0.68,0.66,0.72$, and 0.67 were found, respectively, for SCORE, CUORE, FRS, QRISK2,

M. Sperti · M. A. Deriu

PolitoBIOMed Lab, Department of Mechanical and Aerospace Engineering, Politecnico di Torino,

Torino, Italy

P. Ruscitti · V. Pavlych · R. Giacomelli Rheumatology Unit, Department of Biotechnological and Applied Clinical Sciences, University of L'Aquila, Aquila, Italy

A. Corrado · F. P. Cantatore Rheumatology Clinic, Department of Medical and Surgical Sciences, University of Foggia, Foggia, Italy

G. Di Benedetto

7HC, srl. Via Giovanni Paisiello 55 CAP 00198, Rome, Italy

G. Di Benedetto

Biomedical Research and Innovation Institute of Cádiz (INiBICA), Research Unit, Puerta del Mar University Hospital, University of Cádiz, Cadiz, Spain 
QRISK3, RRS, and ASSIGN. AUC values for the ML algorithms were: 0.70 for SVM, 0.73 for RF, and 0.64 for KNN. Feature analysis showed that C-reactive protein (CRP) has the highest importance, while SBP and hypertension treatment have lower importance.

Conclusions: All of the evaluated CV risk algorithms exhibit a poor discriminative ability, except for RRS and SCORE, which showed a fair performance. For the first time, we demonstrated that AS patients do not show the traditional ones used by CV scores and that the most important variable is CRP. The present study contributes to a deeper understanding of $\mathrm{CV}$ risk in AS, allowing the development of innovative $C V$ risk patient-specific models.

Keywords: Ankylosing Cardiovascular risk; C-reactive protein; Machine learning

\section{DIGITAL FEATURES}

This article is published with digital features to facilitate understanding of the article. To view digital features for this article go to https://doi. org/10.6084/m9.figshare.12887285.

\section{Key Summary Points}

\section{Why carry out this study?}

Cardiovascular disease (CVD) represents an important cause of morbidity and mortality among patients with Ankylosing Spondylitis (AS), so of cardiovascular $(\mathrm{CV})$ risk prediction has a pivotal role in these patients.

The currently available cardiovascular risk algorithms demonstrate only fair or moderate discriminative ability in patients with AS.
In this study, the performance of seven cardiovascular risk predictors is evaluated in a multicentric cohort of AS patients from Italian Rheumatology Units. Moreover, for the first time in literature, performance and calibration of traditional $\mathrm{CV}$ predictors have been here compared with the novel paradigm of machine learning (ML).

\section{What was learned from the study?}

All the CV risk algorithms evaluated exhibit a poor discriminative ability, except for Reynold's Risk Score (RRS) and Systematic Coronary Risk Evaluation (SCORE) which showed a fair performance.

The adaptation of CV risk algorithms according to European League Against Rheumatism (EULAR) recommendations did not provide a significant improvement in discriminative ability.

Patients with AS do not present, among the top features, the traditional ones used by FRS and other traditional methods; the most important variable is $\mathrm{C}$-reactive protein (CRP). This is consistent with the result regarding RRS, which shows the best discriminative ability, probably because it includes CRP as a variable.

Machine-learning algorithms can be helpful in a better cardiovascular assessment in patients with Ankylosing Spondylitis and demonstrate that C-reactive protein can be a key feature of an increased risk in these patients.

Taking into account this variable in future ML studies could increase classification performances on AS patients.

\section{INTRODUCTION}

Ankylosing spondylitis (AS) is a spondyloarthritis (SpA) that deeply affects physical 
function and quality of life, and comorbidities contribute to the prognosis of this disease [1]. Patients with rheumatoid arthritis (RA) and psoriatic arthritis (PsA) show an increased risk of cardiovascular disease (CVD) [2, 3] and traditional CV risk algorithms perform poorly in these populations [4-6]. AS patients show a $20-40 \%$ increase of mortality due to CVD compared to general population [7-9]. Many traditional and disease-related factors contribute to the CVD risk in AS [10-13].

The European League Against Rheumatism (EULAR) recommended that physicians conduct an annual assessment of CV risk in AS patients [14]. The identification of high CV risk AS patients is particularly important in order to improve preventive strategies. Several CV risk algorithms have been proposed over time [15]. The performance and calibration of algorithms for calculating CV risk in RA patients can still be considered a conundrum. In particular, different scores (Framingham Score (FRS), Systematic Coronary Risk Evaluation (SCORE), Reynold's Risk Score (RRS), and QRISK2), tend to underestimate $\mathrm{CV}$ risk in RA patients; the risk observed exceeds the predicted one; the different scores appear slightly calibrated for RA subjects $[16,17]$. EULAR recommended to adapt the general population risk algorithms with a multiplication by the factor of 1.5 in RA patients and other inflammatory arthritis, except for QRISK2, QRISK3, and ASSIGN, which are characterized by a multiplication factor intrinsic to the algorithm for RA [18]. Recently, it has been shown that the above-mentioned risk predictor algorithms provide a less accurate prediction of CV risk in PsA patients compared to general population $[4,5]$.

In this study, the performance of FRS, SCORE, QRISK2, QRISK3, RRS, ASSIGN, and the Italian Progetto CUORE individual score is evaluated in a multicentric cohort of AS patients from Italian Rheumatology Units. Moreover, for the first time in the literature, performance and calibration of traditional $\mathrm{CV}$ predictors have been compared with the novel paradigm of machine learning (ML).

ML was recently introduced in cardiology to face challenges that cannot be solved by traditional statistical methods $[24,25]$. A comparative study between Framingham and quantum neural network-based approach showed how Framingham is outdated and the outstanding potential of ML applied to CV risk prediction [20].

ML belongs to the field of artificial intelligence and it was designed for developing intelligent systems able to learn how to solve a specific problem without being explicitly programmed for it. The learning process is made possible by deriving knowledge from the huge quantity of data present in almost every field (i.e., "big data") and has the objective of making predictions. The two biggest subsets of ML are supervised learning (SL) and unsupervised learning. In the first case, the model is built from a database that already contains the desired output, such as CVD outcome. In the second case, there is no prior knowledge about the event inside the dataset, therefore the model aims at finding subgroups of the original dataset that have common features. ML does not present the same limitations as in the case of traditional statistical methods. Particularly, not many assumptions must be made on the underlying data and non-linearities can be addressed easily. Also, ML can identify hidden variables of a model by inferring them from other variables.

In this work, SL classification approach was adopted to predict the CV risk from a database of AS patients for which the final event was already known.

\section{METHODS}

A retrospective analysis of prospectively collected data from AS cohort of six Italian Rheumatology Units was conducted in November 2018. At baseline (November 2008), patients fulfilled the 1984 Modified New York Criteria and without a personal history of $\mathrm{CV}$ disease (CVD) were consecutively included in this study. Only patients with fully available information allowing the calculation of all the $\mathrm{CV}$ risk at baseline from historical datasets has been recruited.

The study was approved on $19 / 6 / 18$ by the Ethics Committee of University Campus Bio- 
Medico di Roma (approval number: 60/18 OSS), and it was conducted in conformity with the Declaration of Helsinki and its later amendments. Written informed consent for the anonymous use of data was obtained from all participants. Baseline characteristics extracted from the cohort database were: age (years), gender (male/female), weight $(\mathrm{kg})$, height $(\mathrm{cm})$, CRP $(\mathrm{mg} / \mathrm{l})$, erythrocyte sedimentation rate (ESR) $(\mathrm{mm} / \mathrm{h})$, axial arthritis (grade of radiographic sacroiliitis and no-radiographic sacroiliitis $\mathrm{Y} / \mathrm{N})$, peripheral arthritis $(\mathrm{Y} / \mathrm{N})$, Bath Ankylosing Spondylitis Disease Activity Index (BASDAI), Bath Ankylosing Spondylitis Functional Index (BASFI), enthesitis $(\mathrm{Y} / \mathrm{N})$, dactylitis $(\mathrm{Y} / \mathrm{N})$, psoriasis $(\mathrm{Y} / \mathrm{N})$, history of $\operatorname{IBD}(\mathrm{Y} / \mathrm{N})$, history of uveitis $(\mathrm{Y} / \mathrm{N})$, family history of CVD $(\mathrm{Y} / \mathrm{N})$, smoking status (Y/N/previous), hypertension $(\mathrm{Y} / \mathrm{N})$, use of antihypertensive medication $(\mathrm{Y} / \mathrm{N})$, use of statins and aspirin $(\mathrm{Y} / \mathrm{N})$, diabetes mellitus $(\mathrm{Y} / \mathrm{N})$, atrial fibrillation $(\mathrm{Y} / \mathrm{N})$, chronic kidney disease stage IV-V $(\mathrm{Y} / \mathrm{N})$, angina or heart attack in a 1st degree relative $<60$ years $(\mathrm{Y} / \mathrm{N})$, systolic blood pressure (SBP) (mmHg), total cholesterol (mg/dl), and high-density lipoprotein cholesterol (HDL-C) (mg/dl).

The primary outcome was the first CV event (fatal and non-fatal), as reported by electronic patient files. Considered CV events were: sudden cardiac death, coronary artery diseases (CAD) (stable and unstable angina pectoris, myocardial infarction), cerebral vascular accident (CVA), transient ischemic attack (TIA), peripheral artery disease (PAD), and heart failure (HF).

The 10-year general FRS for CVD [21], QRISK2 [22], QRISK3 [23], CUORE [24], RRS [25], and ASSIGN [26] were calculated using already-published algorithms. SCORE algorithm for low-risk countries was used [27]. Cut-off values that mark the difference between low-tointermediate risk and intermediate-to-high risk were $10 \%$ and $20 \%$, respectively, except for SCORE in which cut-off values were $1 \%$ and $5 \%$ and for ASSIGN in which cut-off value that marks the difference between low to high risk is $20 \%$.

The default median value 15.89 for the Scottish Index of Multiple Deprivation (SIMD) was used to calculate the ASSIGN score.
In order to calculate individual risk for $\mathrm{CV}$ within 10 years for all seven algorithms, baseline medical data were used.

The area under the receiver operating characteristic (ROC) curve, which is like the concordance-statistic ( $c$-statistic), was used for the evaluation of the discriminatory ability of all seven algorithms.

The comparison of the agreement between observed and predicted number of CV events in stratified groups by deciles, sextiles, or septiles of the predicted risk, as appropriate, using the Hosmer-Lemeshow (HL) test allowed the assessment of calibration.

Fisher's exact test and Mann-Whitney test were used for analysis of contingency table and comparison between ranks, respectively. Stata V.14 was used for statistical analysis.

\section{Machine Learning}

SL classification was adopted to predict the CV risk from a database of AS patients, for which the final event was already known. SL algorithms contain previous knowledge about data (i.e., labels describing the desired output of the model) and need to be trained using this knowledge before being applied to completely new data. The main goal was to build a model from a dataset (that already contains desired outputs) and to use it to make predictions on future data or data for which desired outputs are not present.

The activity flow of the work was divided in the following phases, typical of ML pipelines:

\section{Phase 1: Training and Validation Database}

Two databases were employed:

1. American patients (3658) from the Framingham Heart Study, retrieved from the Kaggle website (https://www.kaggle.com/ datasets). Risk factors included in this dataset were: gender (0: female, 1: male), age (years), smoking status (0: nonsmoker, 1: smoker), hypertension treatment (0: not treated, 1: treated), total cholesterol $(\mathrm{mg} /$ $\mathrm{dl})$, SBP (mmHg), body mass index (BMI, $\mathrm{kg} /$ 
$\mathrm{m}^{2}$ ), diabetes (0: without diabetes, 1 : with diabetes) and CVD event (0: without CVD, 1: with CVD). In this dataset, 557 patients had a CVD and 3101 did not. This dataset is considered representative for a general population. From now on, this dataset will be indicated as the general dataset.

2. AS patients (133) were included in the study. Some risk factors included in this dataset were the same as those included in the general dataset: gender, age, smoking status, hypertension treatment, total cholesterol, SBP, BMI, diabetes and CVD event. Other non-traditional risk factors were also used: pathology time window (PTW, years), CVD family history (0: no, 1 : yes), atrial fibrillation (AF, 0: no, 1: yes), HDL-C (mg/dl), use of cardio aspirin (0: no, 1: yes), CRP (mg/l), peripheral arthritis (0: no, 1: yes), enthesitis (0: no, 1 : yes), dactylitis ( $0:$ no, $1:$ yes), IBD (0: no, 1: yes), uveitis (0: no, 1: yes), diabetes (0: no, 1: yes), comorbidity ( 0 : no, 1 : yes), use of statins ( 0 : no, 1: yes) and CVD event (0: no, 1: yes). In this dataset, 18 patients had a CVD and 115 did not. From now on, this dataset will be indicated as the AS dataset.

It is worth noting that about $15 \%$ of patients of each database had a CVD event, therefore this is a case of classes' imbalance.

\section{Phase 2: Algorithm Selection and Development}

Three ML techniques were considered to calculate the CV risk: Support vector machine (SVM), random forest (RF), and k-nearest neighbor (KNN) [28-31]. All ML algorithms were developed in Python 3.7.2, with the help of the following scientific computation libraries: NumPy, to manipulate data; Scikit-learn, to implement ML pipelines; Pandas, to manipulate data at a higher level than with NumPy, and Matplotlib, to visualize data.

\section{Phase 3: Dataset Preprocessing and Feature Analysis}

Data were always standardized to take them on the same scale. ML techniques were first applied to the general dataset, using only six traditional features (i.e., age, sex, SBP, total cholesterol, smoking status, and hypertension treatment). The same traditional features were used also on AS dataset to make results comparable with the traditional ones. The general dataset does not contain missing values, while the AS contain several missing values in rheumatic features. Therefore, features with more than 40 missing values were removed, while the others were imputed, because by removing them there was the possibility to remove important information from the dataset or, for small dataset like the one we have, to compromise model's reliability. Imputation followed this strategy: in binary attributes missing values were substituted by 0 (absence of event), while in numeric attribute they were substituted by the normal value over the general Italian population.

In case of continuous features, we imputed the feature from the general database (nonrheumatic patients) and in case of binary features we imputed 0 (= no pathological event). Therefore, in general, we imputed non-recorded features as "non-pathological" thus following a conservative approach toward a more robust model with minimized biases. Moreover, concerning imputation of continuous variables, it is worth saying that, since the AS dataset was very small in size, estimating missing values by the mean value over these patients, might have low statistical significance. Concerning imputation of binary variables, the very vast majority of the patients presented 0 as binary value in those variables. Finally, it is worth mentioning that Framingham features employed to train and test the ML predictor were in the vast majority present for patients in the AS dataset.

\section{Phase 4: Classifier Training and Validation}

To train the classifiers, a balanced dataset was used (i.e., equal number of patients with and without CVD event, about 600 samples). This 
dataset contained patients from the general dataset because they were enough to successfully train an ML algorithm. Then, the classifier was tested on an unbalanced dataset (about $15 \%$ of patients which had a CVD event), composed by the remaining data not used during training (about 3100 samples). Bootstrap technique was used to assess model performance, with 25 random splits with replacement. This sampling technique, by means of the iterative dataset's random splits, gives the possibility to calculate different times algorithms performances on different patients' subsets, making performance evaluation more reliable. The trained model was also validated over the SpA population, i.e., the AS dataset. The models' hyperparameters (SVM: $\mathrm{C}$ in the case of a linear kernel and $C$ and $\gamma$ in the case of a radial basis function kernel; RF: number of trees and splitting criterion; KNN: number of neighbors, i.e., $k$ ) were optimized by means of grid search, setting AUC as the scoring function and performing a fourfold cross validation. ML classifiers presented the following optimized parameters after grid search:

- SVM: radial basis function kernel, $C=0.1$ and $\gamma=0.01$;

- RF: entropy as splitting criterion and 500 trees;

- KNN: Minkowski distance metrics and $K=25$.

\section{Phase 5: Classifier Evaluation Metrics and Evaluation}

Discriminatory ability for the algorithms was assessed by ROC curves and AUC values, sensitivity, specificity, accuracy, positive predictive value, and negative predictive value. Calibration between predicted and observed events was evaluated by Hosmer-Lemeshow tests by comparing the agreement of $\mathrm{CV}$ events in groups stratified in deciles. A comparison between traditional (FRS, CUORE, and SCORE risk scores) and novel techniques (SVM, RF, and $\mathrm{KNN}$ ) was performed to explore performances of traditional risk predictors on AS, with and without EULAR correction coefficient. Performance metrics were calculated for the two cut-offs: low-to-intermediate $(10 \%$ in the case of Framingham and CUORE, 1\% in the case of SCORE) and intermediate-to-high $(20 \%$ in the case of FRS and CUORE, 5\% in the case of SCORE). ML output is binary; therefore, it does not present different cut-off values, but only one threshold (equal to 50\%) used to binarize the output.

First, traditional algorithms (FRS, CUORE, and SCORE) were evaluated on the general population and on the AS database. Second, ML techniques were applied the general population by means of bootstrap technique and performances were compared with FRS as reference for traditional methods. Finally, obtained models trained on the general population were validated on the AS dataset.

Feature importance analysis was performed on AS dataset through importance of RF, pretrained on balanced datasets using all AS features. This step had the aim of evaluating each variable's role and importance as CV risk predictive parameters.

\section{RESULTS}

Data from 133 Caucasian AS patients (1330 patient-years) were analyzed. During follow-up, 18 patients had a CV event (1.35 events per 100 patient/years): eight cases of myocardial infarction, one case of stable angina pectoris, three cases of stroke, two cases of TIA, two cases of PAD, and two cases of HF. No fatal events were reported. The primary outcome was adjusted to fit each CV risk algorithm, leaving 18 for Framingham, 13 for SCORE (38), and $11 \mathrm{CV}$ events for QRISK2, RRS, CUORE, and ASSIGN. As the RRS is not applicable to patients with diabetes or those younger than 45 years, these patients $(n=70)$ were excluded, and only 63 patients were included in the analysis of RRS. Patient's characteristics are summarized in Table 1.

c-statistic scores of 0.71 (95\% CI $0.55-0.87$ ), $0.61 \quad(95 \% \quad$ CI $\quad 0.41-0.81), \quad 0.66 \quad(95 \% \quad$ CI $0.51-0.81), 0.68$ (95\% CI 0.50-0.86), 0.66 (95\% CI 0.48-0.84), 0.72 (95\% CI 0.55-0.89) and 0.67 (95\% CI 0.48-0.86) were found for SCORE, 
Table 1 Patients' characteristics at baseline

\begin{tabular}{|c|c|c|c|c|}
\hline & AS tot $(n=133)$ & $\begin{array}{l}\text { AS without CVD } \\
(n=115)\end{array}$ & $\begin{array}{l}\text { AS with CVD } \\
(n=18)\end{array}$ & $\mathbf{p}$ \\
\hline Age (years) & $46(39-54)$ & $45(38-53)$ & $55(46-64)$ & 0.003 \\
\hline Females (\%) & 45.11 & 46.96 & 33.33 & $\mathrm{Ns}$ \\
\hline Disease duration (years) & $13.34(11.34-17.89)$ & $12.73(11.09-15.93)$ & $16.76(13.34-21.34)$ & 0.007 \\
\hline HLA-B27 (\%) & 42.86 & 43.48 & 38.89 & Ns \\
\hline BASDAI & $4.25(2.62-6.2)$ & $4.1(2.6-5.64)$ & $6.5(4-7.2)$ & 0.04 \\
\hline BASDAI < $4(\%)$ & 50 & 54.67 & 23.08 & 0.06 \\
\hline BASFI & $3(1.25-5.25)$ & $2.5(0.8-4.7)$ & $5.4(2.1-6)$ & 0.03 \\
\hline MRI sacroiliitis (\%) & 90.74 & 90.53 & 92.31 & ns \\
\hline MRI spondylitis (\%) & 34.95 & 32.97 & 50 & ns \\
\hline Syndesmophytes (\%) & 31.62 & 28 & 52.94 & 0.04 \\
\hline Enthesitis (\%) & 32.58 & 31.58 & 38.89 & ns \\
\hline CRP (mg/l) & $3.2(1.6-8)$ & $3(1.36-7)$ & $4.4(3-12.2)$ & 0.03 \\
\hline $\operatorname{ESR}(\mathrm{mm} / \mathrm{h})$ & $21(11-32)$ & $19.5(11-31)$ & $24(12-45)$ & ns \\
\hline Smokers (\%) & 33.83 & 32.17 & 44.44 & ns \\
\hline CVD family history (\%) & 27.07 & 24.35 & 44.44 & Ns \\
\hline Atrial fibrillation (\%) & 1.50 & 0.87 & 5.56 & ns \\
\hline Diabetes (\%) & 7.52 & 6.96 & 11.11 & ns \\
\hline Stage 3-5 of chronic kidney disease (\%) & 1.50 & 0 & 11.11 & 0.02 \\
\hline Migraine (\%) & 12.03 & 13.91 & 0 & ns \\
\hline Antipsychotics (\%) & 0.75 & 0.87 & 0 & ns \\
\hline Systolic blood pressure ( $\mathrm{mmHg}$ ) & $125(120-140)$ & $127.5(120-135)$ & $125(120-140)$ & ns \\
\hline SCORE & $1(0-2)$ & $0(0-1)$ & $2(1-3)$ & 0.001 \\
\hline CUORE & $1.54(0.06-4.54)$ & $1.06(0.06-4)$ & $5.01(2.01-8.06)$ & 0.034 \\
\hline FRS & $6.53(2.08-13.53)$ & $6.01(2.07-12.03)$ & $13.06(8.03-17.07)$ & 0.037 \\
\hline QRISK2 & $4.01(1.01-10.06)$ & $3.06(1-8.03)$ & $12.06(6.01-17)$ & 0.003 \\
\hline QRISK3 & $5.1(1.4-11.4)$ & $4.6(1.1-9.45)$ & $12(3.5-17.1)$ & 0.006 \\
\hline RRS & $3(1-5)$ & $3(1-5)$ & $9(4-12)$ & $<0.001$ \\
\hline ASSIGN & $6(3-12)$ & $6(3-10)$ & $13(6-18)$ & 0.003 \\
\hline
\end{tabular}

Data are expressed as median (25-75th percentile), unless otherwise indicated

Bold numbers indicate significant $\mathrm{p}$-values

$A S$ ankylosing spondylitis, BASDAI Bath Ankylosing Spondylitis Disease Activity Index, BASFI Bath Ankylosing Spondylitis Functional Index, CRP C-reactive protein, $C V D$ cardiovascular disease, FRS Framingham Risk Score, $M R I$ magnetic resonance imaging, ESR erythrocyte sedimentation rate, $R R S$ Reynold's Risk Score, SCORE Systematic Coronary Risk Evaluation 
CUORE, FRS, QRISK2, QRISK3, RRS, and ASSIGN, respectively (Fig. 1a-g).

Overall, the multiplicative factors did not seem to improve the performances of any of the algorithms: $c$-statistic scores of 0.71 (95\% CI 0.52-0.87), 0.63 (95\% CI 0.44-0.83), 0.66 (95\% CI $0.51-0.81), 0.68$ (95\% CI $0.49-0.86), 0.66$ (95\% CI $0.48-0.83$ ), 0.72 (95\% CI $0.55-0.89$ ) and 0.65 (95\% CI $0.46-0.85)$ were found for SCORE*1.5 $\left(p=\mathrm{ns}\right.$ vs. SCORE), CUORE ${ }^{*} 1.5$ ( $p=\mathrm{ns}$ vs. CUORE), $\mathrm{FRS}^{\star} 1.5(p=\mathrm{ns}$ vs. FRS), QRISK2-RA ( $p=\mathrm{ns}$ vs. QRISK2), QRISK3-RA ( $p=$ ns vs. QRISK3), RRS ${ }^{\star} 1.5$ ( $p=$ ns vs. RRS) and ASSIGN-RA ( $p=$ ns vs. ASSIGN), respectively (Fig. 1h-p).

Calibration plots are reported in Fig. 2a-p. The Hosmer-Lemeshow test did not demonstrate a poor model for any of the $\mathrm{CV}$ risk algorithms: SCORE $p=0.6$; $\operatorname{SCORE}^{*} 1.5 p=0.6$; CUORE $p=0.54 ;$ CUORE ${ }^{\star} 1.5 p=0.32 ;$ FRS $p=0.74 ; \mathrm{FRS}^{*} 1.5 p=0.69 ; \mathrm{RRS} p=0.74 ; \mathrm{RRS}^{*} 1.5$ $p=0.38 ; \quad$ QRISK2 $p=0.079 ; \quad$ QRISK2-RA $p=0.45$; QRISK3 $p=0.15$; QRISK3-RA $p=0.15$; ASSIGN $p=0.8$; ASSIGN-RA $p=0.25$. Overall, we found a trend towards increased CV events compared to the expected, especially at lowand middle-risk levels.

Sensitivity and specificity of the $10 \%$ and 20\% cut-off points for CV risk for FRS, QRISK2, QRISK3, CUORE, RRS, and ASSIGN and of the $1 \%$ and $5 \%$ cut-off points for CV risk for SCORE are reported in Table 2 .

ML techniques were applied on AS patients. Models were built on the general population and validated over the rheumatic one. ML was trained with only six Framingham features (gender, age, SBP, hypertension treatment, smoking status, and total cholesterol). AUC values in the case of AS population are: 0.70 (95\% CI 0.55-0.85) for SVM, 0.73 (95\% CI 0.61-0.85) for RF and 0.64 (95\% CI 0.50-0.77) for $\mathrm{KNN}$. The correspondent ROC curves are reported in (Fig. 3a-c).

Overall, calibration plots demonstrated that observed CV risk is lower than the predicted one (Fig. 3d-f). Sensitivity, specificity, accuracy, positive predictive value, and negative predictive value are reported in Table 3 .

Feature analysis was performed in this study by means of RF's importance. RF was pre-trained in an AS dataset using all rheumatic features and it ranked features based on their relative importance. Results are represented in Fig. 3g. It is evident from the plot that in the case of AS, CRP has the highest importance, while SBP and hypertension treatment have lower importance. Feature importance analysis might be crucial to select variables to be included in further risk predictor development.

Concerning ML algorithms, a single threshold equal to $50 \%$ necessary to binarize the output (subjects above the threshold are considered at risk of developing a CVD) has been used instead of considering a cut-off, which is in the ML case meaningless. Therefore, among patients with $\mathrm{CV}$ events, $22.2 \%$ were under the threshold according to KNN, $27.8 \%$ were under the threshold according to SVM, and $27.8 \%$ were under the threshold according to RF.

\section{DISCUSSION}

CVD represents an important cause of morbidity and mortality among patients with AS [32]. Only an accurate prediction of CV risk in these patients can allow the achievement of preventive strategies. All of the $\mathrm{CV}$ risk algorithms evaluated in the present study exhibit a poor discriminative ability, except for RRS and SCORE, which showed a fair performance. Intriguingly, only RRS includes CRP as a key variable in the assessment of $\mathrm{CV}$ risk. The adaptation of CV risk algorithms according to EULAR recommendations did not provide a significant improvement in discriminative ability. Notably, the Hosmer-Lemeshow test did not demonstrate a poor model fit for any of the $\mathrm{CV}$ algorithms considered in the present study; this is probably due to a low power of the test because of a small sample size. Regarding the cut-off point that marks the difference between low- and medium-high risk and the difference between low-medium and high risk, FRS* 1.5 showed the best sensitivity, $76.47 \%$ (95\% CI 50.1-93.19) and 52.94\% (95\% CI 6.02-60.97), respectively, and CUORE the best specificity, 93.58\% (95\% CI 87.22-97.38) and 99.08\% (95\% CI 93.53-99.98). The incidence of CVD in the present cohort may appear slightly increased as 

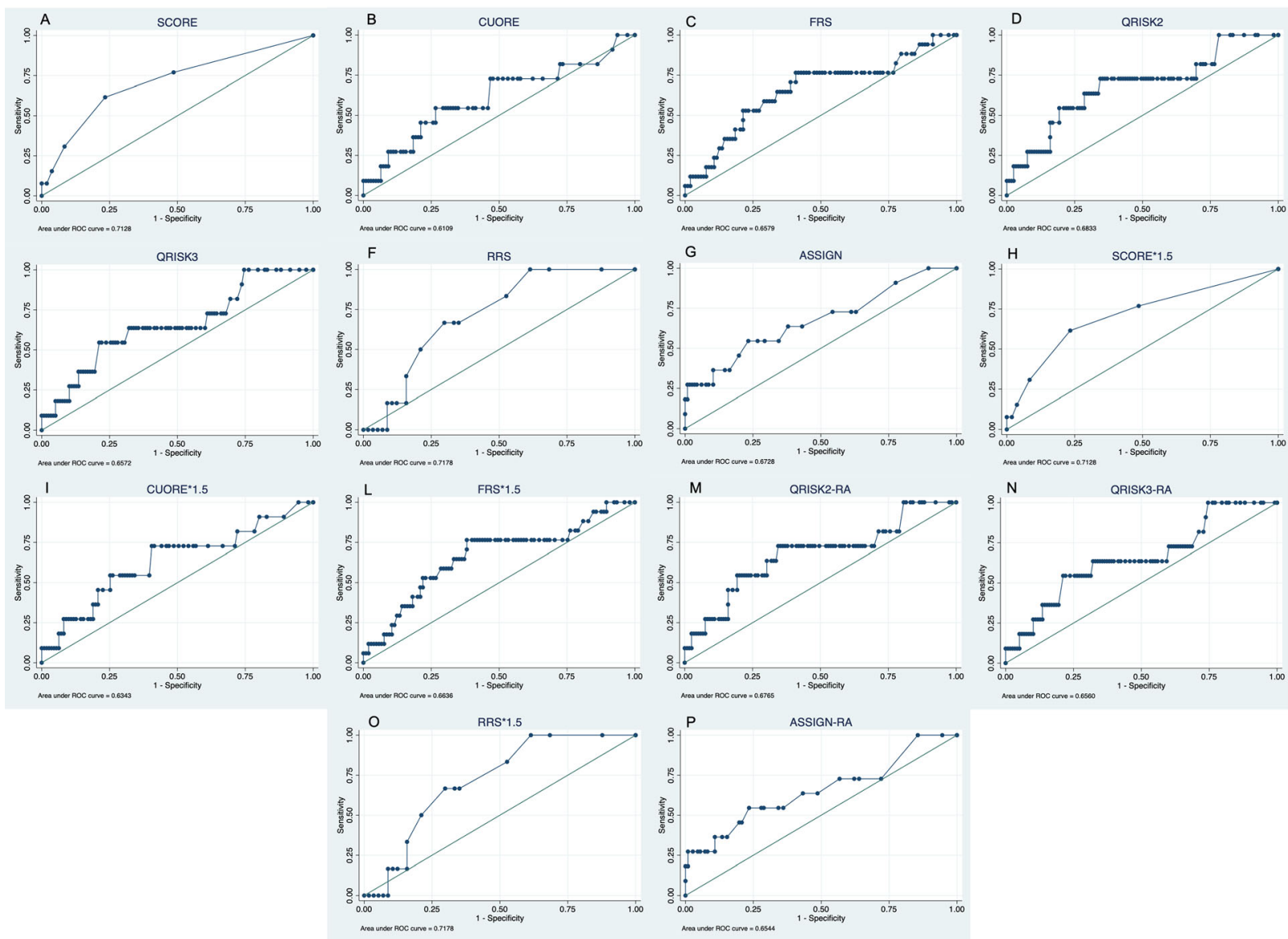

Fig. 1 ROC curves of traditional cardiovascular risk algorithms. $c$-statistics scores: 0.71 (95\% CI $0.52-0.87$ ), 0.61 (95\% CI 0.41-0.81), 0.66 (95\% CI 0.51-0.81), 0.68 (95\% CI 0.50-0.86), 0.66 (95\% CI 0.48-0.84), 0.72 (95\% CI $0.55-0.89$ ), 0.67 (95\% CI $0.48-0.86$ ), 0.71 (95\% CI $0.52-0.87$ ), 0.63 (95\% CI $0.44-0.83$ ), 0.66 (95\% CI $0.51-0.81), \quad 0.68$ (95\% CI $0.49-0.86), 0.66$ (95\% CI $0.48-0.83$ ), 0.72 (95\% CI $0.55-0.89$ ) and 0.65 (95\% CI

compared with other studies [33-37]; it should be noted that in the present study, a broad spectrum of CVDs has been taken into account, including TIA, PAD, and HF. Broadly, the analysis of the present study demonstrated the huge limitations of both traditional and adapted according to EULAR recommendations CV risk algorithms in patients with AS. Therefore, an ML approach has been carried out to better predict $\mathrm{CV}$ risk in these patients.

Considering the lack of substantial differences in performance among the algorithms
0.46-0.85) for SCORE (a), CUORE (b), FRS (c), QRISK2 (d), QRISK3 (e), RRS (f), ASSIGN (g), SCORE*1.5 (h), CUORE*1.5 (i), FRS*1.5 (l), QRISK2RA (m), QRISK3-RA (n), RRS*1.5 (o), and ASSIGN-RA (p)

examined in the present study and the availability of data from patients retrieved from the Kaggle website, we chose FRS as the reference for comparison in ML analysis. AS patients had $58.82 \%$ sensitivity and $66.99 \%$ specificity with the cut-off that marks the difference between low and medium-high risk and $25.53 \%$ sensitivity and $89.32 \%$ specificity with the cut-off that marks the difference between low-medium and high risk. Therefore, sensitivity drastically decreased when predicting CV risk in AS patients and the EULAR multiplication factor 

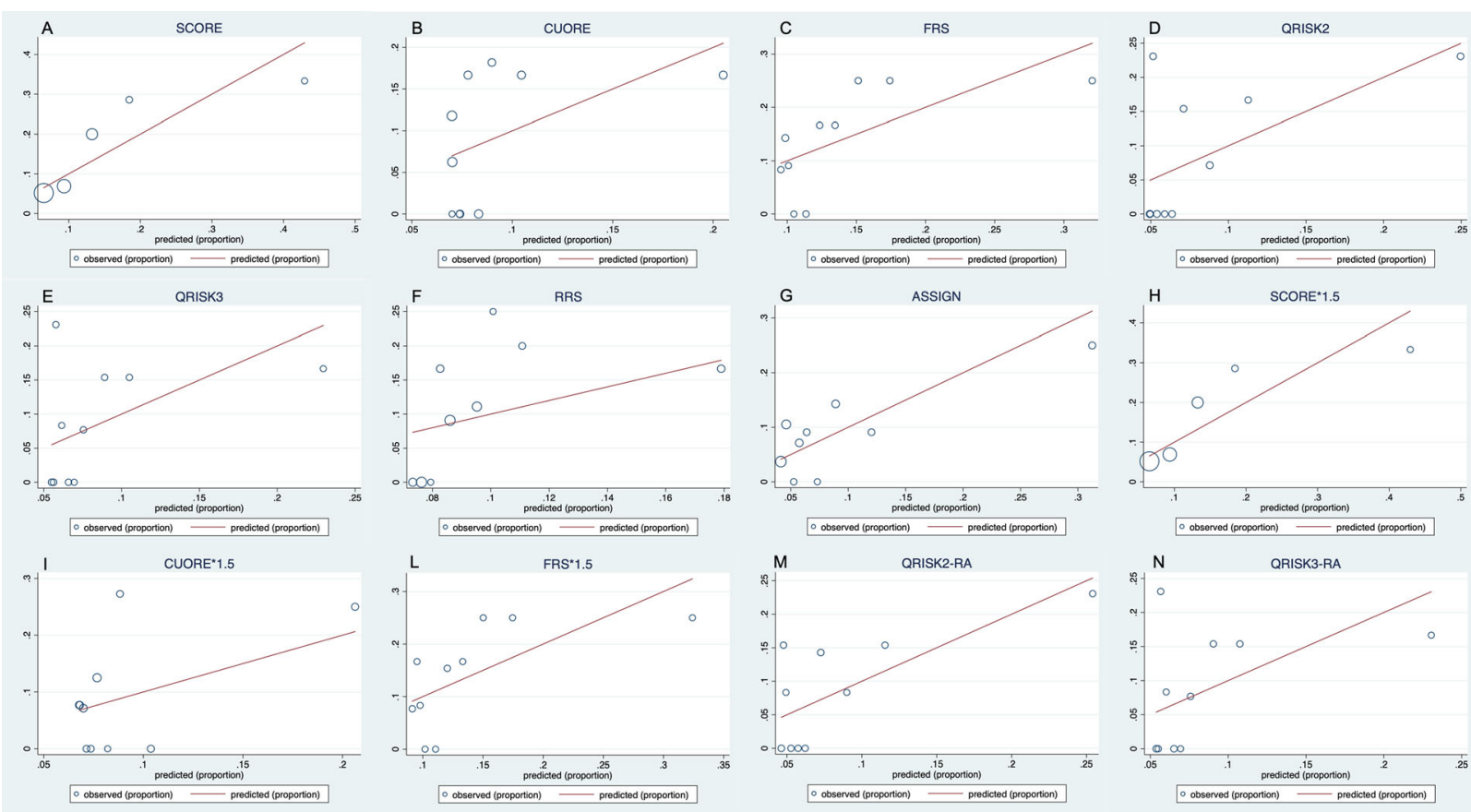

H SCORE* 1.5
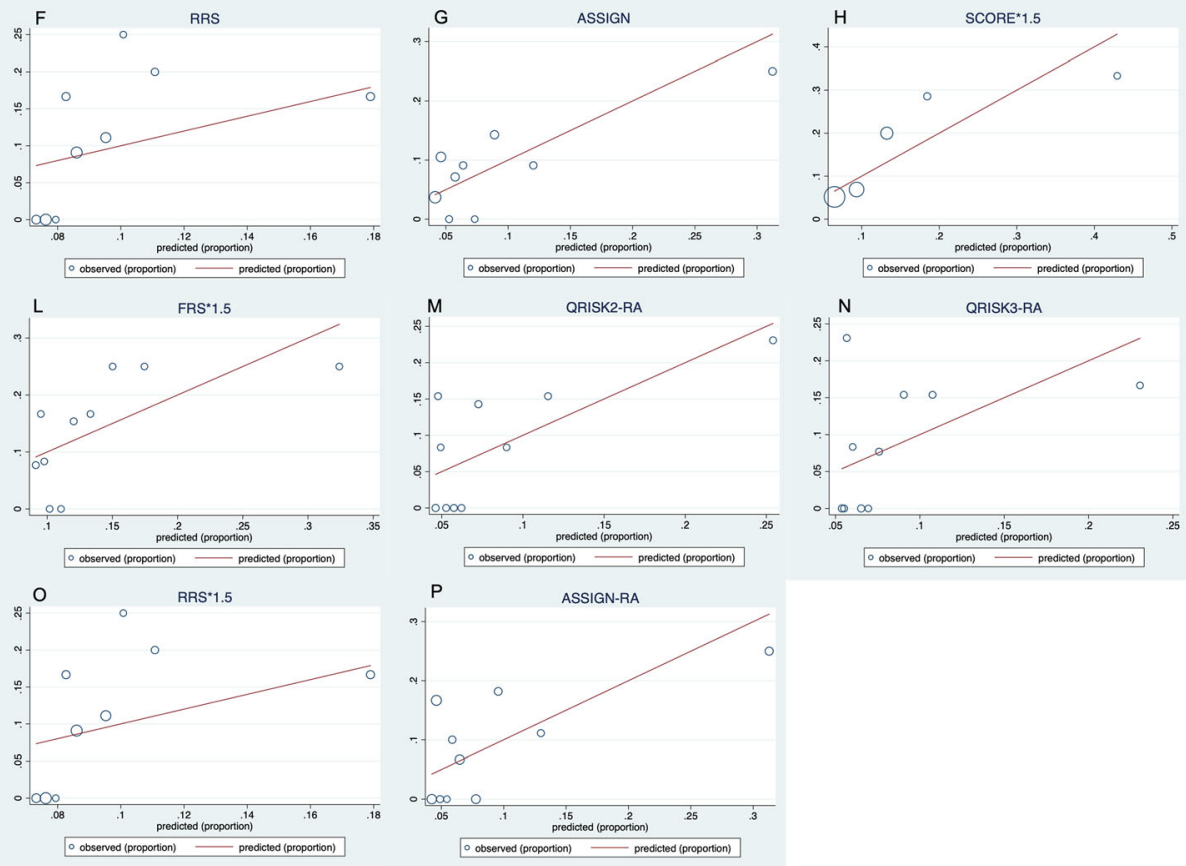

Fig. 2 Calibration plots comparing observed vs. predicted risk for SCORE (a), CUORE (b), FRS (c), QRISK2 (d), QRISK3 (e), RRS (f), ASSIGN (g), SCORE*1.5 (h),

CUORE*1.5 (i), FRS*1.5 (1), QRISK2-RA (m), QRISK3RA (n), RRS*1.5 (o), and ASSIGN-RA (p)

did not present an acceptable improvement. These results lead to hypothesize that an increase in sensitivity occurs with a correspondent reduction of specificity when growing the cut-off. However, accuracy remained the same or slightly decreased. EULAR multiplication factor acts in a similar way to the cut-off strategy.

For this reason, we explored the application of ML methods as new CV risk models for rheumatic patients. The general dataset was used to evaluate ML performances and to develop stable models, thanks to the large number of patients contained in it. Better results were obtained using a balanced dataset (i.e., with the same number of patients who experienced a CV event and patients who do not) to train the ML algorithms, since ML

methods learn from data, therefore if the input data is asymmetric, the model will learn a consequent asymmetric decision rule. Parameter optimization has always been performed only on training datasets, otherwise performances would have been too optimistic. SVM has $66.67 \%$ sensitivity and $60 \%$ specificity; RF $61.11 \%$ sensitivity and $66.09 \%$ specificity, and KNN $77.78 \%$ sensitivity and $54.78 \%$ specificity. These results outperformed sensitivity with respect to FRS, but they tend to overestimate the risk. Therefore, future work is necessary to overcome this limitation.

Recently, it has been demonstrated that deep belief networks performed better than other prediction methods on $\mathrm{CV}$ risk assessment using six variables (age, SBP, diastolic blood pressure, HDL cholesterol, smoking status, and 
Table 2 Sensitivity, specificity, positive predictive value (PPV), negative predictive value (NPV), and accuracy of cut-off values in traditional and adapted according to EULAR recommendations CV risk scores

\begin{tabular}{|c|c|c|c|c|c|}
\hline Variable & Sensitivity (\%) & Specificity (\%) & PPV (\%) & NPV (\%) & Accuracy (\%) \\
\hline SCORE > 1\% & $\begin{array}{l}76.92 \\
\quad(46.19-94.96)\end{array}$ & $\begin{array}{l}51.40 \\
\quad(41.54-61.18)\end{array}$ & $\begin{array}{l}16.13 \\
\quad(11.87-21.54)\end{array}$ & $\begin{array}{l}94.83 \\
\quad(86.98-98.05)\end{array}$ & $\begin{array}{l}54.17 \\
\quad(44.83-63.29)\end{array}$ \\
\hline CUORE $>10 \%$ & $\begin{array}{l}18.18 \\
\quad(2.28-51.78)\end{array}$ & $\begin{array}{l}93.58 \\
\quad(87.22-97.38)\end{array}$ & $\begin{array}{l}22.22 \\
\quad(6.32-54.77)\end{array}$ & $\begin{array}{l}91.89 \\
\quad(89.52-93.77)\end{array}$ & $\begin{array}{l}86.67 \\
\quad(79.25-92.18)\end{array}$ \\
\hline Qrisk2 > 10\% & $\begin{array}{l}54.55 \\
\quad(23.38-83.25)\end{array}$ & $\begin{array}{l}76.47 \\
\quad(67.82-83.76)\end{array}$ & $\begin{array}{l}17.65 \\
\quad(10.25-28.68)\end{array}$ & $\begin{array}{l}94.79 \\
\quad(90.43-97.23)\end{array}$ & $\begin{array}{l}74.62 \\
\quad(66.24-81.84)\end{array}$ \\
\hline Qrisk3 > 10\% & $\begin{array}{l}54.55 \\
\quad(23.38-83.25)\end{array}$ & $\begin{array}{l}73.73 \\
\quad(64.83-81.40)\end{array}$ & $\begin{array}{l}16.22 \\
\quad(9.44-26.43)\end{array}$ & $\begin{array}{l}94.57 \\
\quad(90.03-97.10)\end{array}$ & $\begin{array}{l}72.09 \\
\quad(63.52-69.73)\end{array}$ \\
\hline FRS $>10 \%$ & $\begin{array}{l}58.82 \\
\quad(32.92-81.56)\end{array}$ & $\begin{array}{l}66.99 \\
\quad(57.03-75.94)\end{array}$ & $\begin{array}{l}22.73 \\
\quad(15.35-32.3)\end{array}$ & $\begin{array}{l}90.79 \\
\quad(84.61-94.65)\end{array}$ & $\begin{array}{l}65.83 \\
\quad(56.62-74.24)\end{array}$ \\
\hline RRS > 10\% & $\begin{array}{l}33.33 \\
\quad(4.33-77.72)\end{array}$ & $\begin{array}{l}84.21 \\
\quad(72.13-92.52)\end{array}$ & $\begin{array}{l}18.18 \\
\quad(5.82-44.44)\end{array}$ & $\begin{array}{l}92.31 \\
\quad(87.08-95.53)\end{array}$ & $\begin{array}{l}79.37 \\
\quad(67.30-88.53)\end{array}$ \\
\hline SCORE $1.5>1 \%$ & $\begin{array}{l}76.92 \\
\quad(46.19-94.96)\end{array}$ & $\begin{array}{l}51.40 \\
\quad(41.54-61.18)\end{array}$ & $\begin{array}{l}16.13 \\
\quad(11.87-21.54)\end{array}$ & $\begin{array}{l}94.83 \\
\quad(86.98-98.05)\end{array}$ & $\begin{array}{l}54.17 \\
\quad(44.83-63.29)\end{array}$ \\
\hline $\begin{array}{l}\text { CUORE } \\
1.5>10 \%\end{array}$ & $\begin{array}{l}27.27 \\
\quad(6.02-60.97)\end{array}$ & $\begin{array}{l}82.88 \\
\quad(74.57-89.37)\end{array}$ & $\begin{array}{l}13.64 \\
\quad(5.24-31.05)\end{array}$ & $92(88.8-94.34)$ & $\begin{array}{l}77.87 \\
\quad(69.46-84.88)\end{array}$ \\
\hline $\begin{array}{l}\text { Qrisk2- } \\
\qquad \text { RA > 10\% }\end{array}$ & $\begin{array}{l}63.64 \\
\quad(30.79-89.07)\end{array}$ & $\begin{array}{l}69.75 \\
\quad(60.65-77.83)\end{array}$ & $\begin{array}{l}16.28 \\
\quad(10.33-24.71)\end{array}$ & $\begin{array}{l}95.40 \\
\quad(90.39-97.86)\end{array}$ & $\begin{array}{l}69.23 \\
\quad(60.54-77.02)\end{array}$ \\
\hline $\begin{array}{l}\text { Qrisk3- } \\
\qquad \text { RA > 10\% }\end{array}$ & $\begin{array}{l}54.55 \\
\quad(23.38-83.25)\end{array}$ & $\begin{array}{l}70.34 \\
\quad(61.23-78.39)\end{array}$ & $\begin{array}{l}14.63 \\
\quad(8.55-23.93)\end{array}$ & $\begin{array}{l}94.32 \\
\quad(89.58-96.97)\end{array}$ & $\begin{array}{l}68.99 \\
\quad(60.25-77.84)\end{array}$ \\
\hline FRS $1.5>10 \%$ & $\begin{array}{l}76.47 \\
\quad(50.10-93.19)\end{array}$ & $\begin{array}{l}55.24 \\
\quad(45.22-64.95)\end{array}$ & $\begin{array}{l}21.67 \\
\quad(16.47-27.96)\end{array}$ & $\begin{array}{l}93.55 \\
\quad(85.82-97.20)\end{array}$ & $\begin{array}{l}58.20 \\
\quad(48.93-67.06)\end{array}$ \\
\hline RRS $1.5>10 \%$ & $\begin{array}{l}66.67 \\
\quad(22.28-95.67)\end{array}$ & $\begin{array}{l}66.67 \\
\quad(52.94-78.6)\end{array}$ & $\begin{array}{l}17.39 \\
\quad(9.69-29.24)\end{array}$ & $95(85.79-98.35)$ & $\begin{array}{l}66.67 \\
\quad(53.66-78.05)\end{array}$ \\
\hline SCORE $>5 \%$ & $\begin{array}{l}15.38 \\
\quad(1.92-45.45)\end{array}$ & $\begin{array}{l}96.26 \\
\quad(90.7-98.97)\end{array}$ & $33.33(9.2-71.17)$ & $\begin{array}{l}90.35 \\
\quad(88.1-92.21)\end{array}$ & $87.5(80.22-92.83)$ \\
\hline CUORE > 20\% & $9.09(0.23-41.28)$ & $\begin{array}{l}99.08 \\
\quad(94.99-99.98)\end{array}$ & $50(6.29-93.71)$ & $\begin{array}{l}91.53 \\
\quad(89.95-92.87)\end{array}$ & $\begin{array}{l}90.83 \\
\quad(84.19-95.33)\end{array}$ \\
\hline Qrisk2 > 20\% & $\begin{array}{l}18.18 \\
\quad(2.28-51.78)\end{array}$ & $\begin{array}{l}92.44 \\
\quad(86.13-96.48)\end{array}$ & $\begin{array}{l}18.18 \\
\quad(5.18-47.45)\end{array}$ & $\begin{array}{l}92.44 \\
\quad(90.20-94.19)\end{array}$ & $86.15(79-91.58)$ \\
\hline Qrisk3 > 20\% & $\begin{array}{l}18.18 \\
\quad(2.28-51.78)\end{array}$ & $\begin{array}{l}92.37 \\
\quad(86.01-96.45)\end{array}$ & $\begin{array}{l}18.18 \\
\quad(5.18-47.45)\end{array}$ & $\begin{array}{l}92.37 \\
\quad(90.12-94.14)\end{array}$ & $\begin{array}{l}86.05 \\
\quad(78.85-91.52)\end{array}$ \\
\hline FRS $>20 \%$ & $25.53(6.81-49.9)$ & $\begin{array}{l}89.32 \\
\quad(81.69-94.55)\end{array}$ & $\begin{array}{l}26.67 \\
\quad(11.56-50.28)\end{array}$ & $\begin{array}{l}87.62 \\
\quad(84.35-90.28)\end{array}$ & $80(71.72-86.75)$ \\
\hline RRS $>20 \%$ & $0(0-45.93)$ & $92.98(83-98.05)$ & 0 & $\begin{array}{l}89.83 \\
\quad(89.16-90.46)\end{array}$ & $\begin{array}{l}84.13 \\
\quad(72.74-92.12)\end{array}$ \\
\hline
\end{tabular}


Table 2 continued

\begin{tabular}{lccccc}
\hline Variable & Sensitivity (\%) & Specificity (\%) & PPV (\%) & NPV (\%) & Accuracy (\%) \\
\hline ASSIGN $>$ 20\% & 27.27 & 91.38 & $23.08(8.82-48.21)$ & 92.98 & 85.83 \\
& $(6.02-60.97)$ & $(84.72-95.79)$ & & $(90.18-95.03)$ & $(78.53-91.38)$ \\
SCORE $1.5>5 \%$ & 15.38 & 96.26 & $33.33(9.2-71.17)$ & 90.35 & $87.5(80.22-92.83)$ \\
& $(1.92-45.45)$ & $(90.7-98.97)$ & & $(88.1-92.21)$ & \\
CUORE & $9.09(0.23-41.28)$ & 96.40 & $20(2.96-67.16)$ & 91.45 & $88.52(81.5-93.58)$ \\
$1.5>20 \%$ & & $(91.03-99.01)$ & & $(89.84-92.83)$ & \\
Qrisk2- & 27.27 & 89.08 & 18.75 & 92.98 & 83.85 \\
RA $>$ 20\% & $(6.02-60.97)$ & $(82.04-94.05)$ & $(7.18-40.77)$ & $(90.17-95.03)$ & $(76.37-89.71)$ \\
Qrisk3- & 27.27 & 89.83 & $20(7.65-42.99)$ & 92.98 & $84.5(77.08-90.27)$ \\
RA > 20\% & $(6.02-60.97)$ & $(82.91-94.63)$ & & $(90.18-95.03)$ & \\
FRS 1.5 > 20\% & 52.94 & 78.10 & 28.13 & 91.11 & 74.59 \\
& $(27.81-77.02)$ & $(68.97-85.58)$ & $(18.04-41.03)$ & $(85.97-94.49)$ & $(65.91-82.04)$ \\
RRS 1.5 > 20\% & 16.67 & 91.23 & $16.67(2.7-59.05)$ & 91.23 & $84.13(72.74-92.12)$ \\
& $(0.42-64.12)$ & $(80.70-97.09)$ & & $(87.81-93.75)$ & \\
ASSIGN- & 36.36 & 89.19 & $25(11.45-46.26)$ & 93.4 & 84.43 \\
RA > 20\% & $(10.93-69.21)$ & $(81.88-94.29)$ & & $(90.01-95.69)$ & $(76.75-90.36)$ \\
\hline D & & &
\end{tabular}

Data are expressed as percentage

FRS Framingham Score, SCORE Systematic Coronary Risk Evaluation; RRS Reynold's Risk Score

diabetes) [38]. SVM had 100\% specificity, 71.8\% sensitivity, and $71.8 \%$ accuracy, hence it was effective in identifying low risk, but it could not correctly predict high risk. RF had $61.4 \%$ specificity, $82.2 \%$ sensitivity, and $77.2 \%$ accuracy. Statistical deep belief networks outperformed all methods, with $73.3 \%$ specificity, $87.6 \%$ sensitivity, and $83.9 \%$ accuracy. However, a better result was obtained by Narain and coworkers who did a comparison between FRS and quantum neural network-based approach, with 98.57\% accuracy [20]. This result shows that different ML methods could be used in CV risk prediction and potentially in the specific field of rheumatic patients.

Future models should also consider the great number of variables associated with $\mathrm{CV}$ risk in rheumatic patients. For this purpose, RF could represent a useful technique to better understand the variables most informative within a bigger dataset. Unfortunately, this method does not consider the possible correlation between two variables. In the present study, we demonstrated that patients with AS do not present, among the top features, the traditional ones used by FRS and other traditional methods; the most important variable is CRP. This is consistent with the result regarding RRS, which shows the best discriminative ability, probably because it includes CRP as a variable. Moreover, CRP plays a key role in AS, as reported elsewhere [39]. Taking into account this variable in future ML studies could increase classification performances on AS patients.

Several weaknesses of this study should be considered. The main limitation of this study is the dataset's dimensions. Basing on this preliminary study, we suggest that a dataset of about 500 or 1000 patients (15\% CV events) might be enough to allow training and validation of solid ML algorithms specific for AS. Moreover, RRS calculation was possible only in 

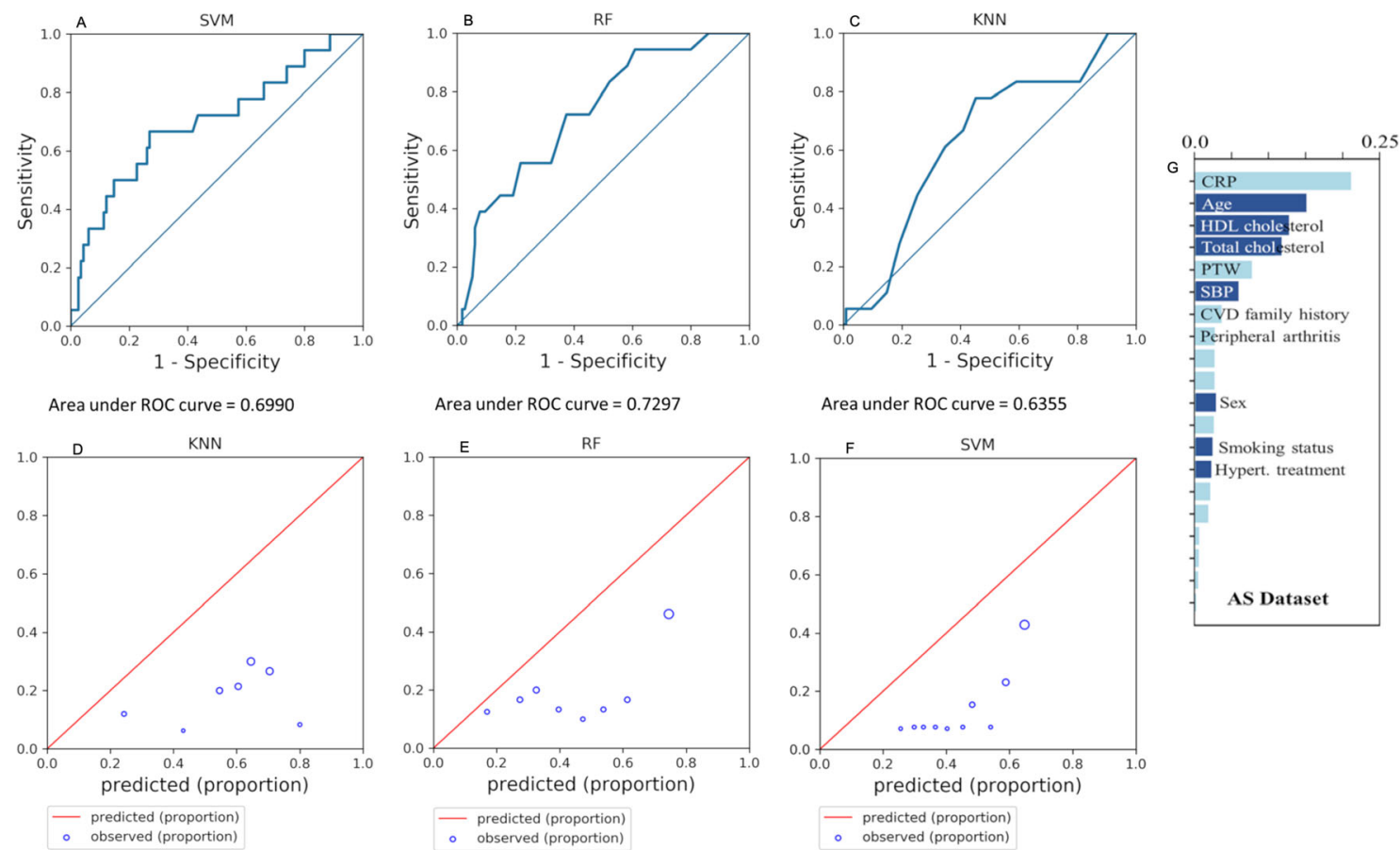

for KNN (d), RF (e), and SVM (f). g Random forest's

Fig. 3 ROC curves of machine learning-based cardiovascular risk algorithms. $c$-Statistics scores: 0.70 (95\% CI 0.55-0.85) for SVM (a), 0.73 (95\% CI 0.61-0.85) for RF importance

(b), and 0.64 (95\% CI $0.50-0.77)$ for $\mathrm{KNN} \mathrm{(c).}$

Calibration plots comparing observed vs. predicted risk

Table 3 Sensitivity, specificity, positive predictive value (PPV), negative predictive value (NPV), and accuracy of cut-off values in machine-learning CV risk scores

\begin{tabular}{llllll}
\hline Variable & Sensitivity (\%) & Specificity (\%) & PPV (\%) & NPV (\%) & Accuracy (\%) \\
\hline KNN & $77.78(52.36-93.59)$ & $54.78(45.23-64.08)$ & $21.21(11.35-31.08)$ & $94.03(88.36-99.7)$ & $57.89(49.03-66.4)$ \\
SVM & 66.67 & 60 & 20.69 & 92 & 60.90 \\
& $(40.99-86.66)$ & $(50.45-69.02)$ & $(10.26-31.11)$ & $(85.86-98.14)$ & $(52.07-69.24)$ \\
RF & 61.11 & 66.09 & 22 & 91.57 & 65.41 \\
& $(35.75-82.70)$ & $(56.67-74.65)$ & $(10.52-33.48)$ & $(85.59-97.54)$ & $(56.68-73.44)$ \\
\hline
\end{tabular}

Data are expressed as percentage

$K N N k$-nearest neighbor, $R F$ random forest, $S V M$ support vector machine

63 patients. Furthermore, only Caucasic Italian patients have been enrolled in the present study. In addition, no information about the possible role of the different medications approved for AS on CVD can be inferred.

\section{CONCLUSIONS}

The present study contributes to a deeper understanding of $\mathrm{CV}$ risk in AS, with a particular focus on $\mathrm{CV}$ risk algorithms. Despite the 
small sample size, it can be concluded that RRS and SCORE has a fair performance in predicting CVD and it can be hypothesized that CRP might play a pivotal role in patients with AS. Moreover, ML could allow the development of innovative patient-specific CV risk models.

\section{ACKNOWLEDGEMENTS}

We thank the participants of the study.

Funding. No funding or sponsorship was received for this study or publication of this article.

Authorship. All named authors meet the International Committee of Medical Journal Editors (ICMJE) criteria for authorship for this article, take responsibility for the integrity of the work as a whole, and have given their approval for this version to be published.

Disclosures. Luca Navarini, Francesco Caso, Luisa Costa, Damiano Currado, Liliana Stola, Fabio Perrotta, Lorenzo Delfino, Michela Sperti, Marco Deriu, Piero Ruscitti, Viktoriya Pavlych, Addolorata Corrado, Giacomo Di Benedetto, Marco Tasso, Massimo Ciccozzi, Alice Laudisio, Claudio Lunardi, Francesco Paolo Cantatore, Roberto Giacomelli, and Antonella Afeltra have nothing to disclose. Ennio Lubrano and Raffaele Scarpa are members of the journal's Editorial Board.

Compliance with Ethics Guidelines. The study was approved on 19/6/18 by the Ethics committee of University Campus Bio-Medico di Roma (approval number: 60/18 OSS), and it was conducted in conformity with the Declaration of Helsinki and its later amendments. Written informed consent was obtained from all participants, if indicated.

Data Availability. The datasets generated during and/or analyzed during the current study are available from the corresponding author on reasonable request.
Open Access. This article is licensed under a Creative Commons Attribution-NonCommercial 4.0 International License, which permits any non-commercial use, sharing, adaptation, distribution and reproduction in any medium or format, as long as you give appropriate credit to the original author(s) and the source, provide a link to the Creative Commons licence, and indicate if changes were made. The images or other third party material in this article are included in the article's Creative Commons licence, unless indicated otherwise in a credit line to the material. If material is not included in the article's Creative Commons licence and your intended use is not permitted by statutory regulation or exceeds the permitted use, you will need to obtain permission directly from the copyright holder. To view a copy of this licence, visit http://creativecommons.org/licenses/by$\mathrm{nc} / 4.0 /$.

\section{REFERENCES}

1. Wang R, Ward MM. Epidemiology of axial spondyloarthritis: an update. Curr Opin Rheumatol. 2018;30:137-43.

2. England BR, Thiele GM, Anderson DR, Mikuls TR. Increased cardiovascular risk in rheumatoid arthritis: mechanisms and implications. BMJ. 2018;361: k1036.

3. Eder L, Harvey P. Cardiovascular morbidity in psoriatic arthritis: what is the effect of inflammation? J Rheumatol. 2017;44:1295-7.

4. Navarini L, Margiotta DPE, Caso F, Currado D, Tasso M, Angeletti S, et al. Performances of five risk algorithms in predicting cardiovascular events in patients with Psoriatic Arthritis: an Italian bicentric study. PLoS One. 2018;13:e0205506.

5. Navarini L, Margiotta DPE, Costa L, Currado D, Tasso M, Angeletti S, et al. Performance and calibration of the algorithm ASSIGN in predicting cardiovascular disease in Italian patients with psoriatic arthritis. Clin Rheumatol. 2019;38:971-6.

6. Arts EEA, Popa C, Den Broeder AA, Semb AG, Toms $\mathrm{T}$, Kitas GD, et al. Performance of four current risk algorithms in predicting cardiovascular events in patients with early rheumatoid arthritis. Ann Rheum Dis. 2015;74:668-74. 
7. Shen J, Shang Q, Tam LS. Targeting inflammation in the prevention of cardiovascular disease in patients with inflammatory arthritis. Transl Res. 2016;167:138-51.

8. Castañeda S, Nurmohamed MT, González-Gay MA. Cardiovascular disease in inflammatory rheumatic diseases. Best Pract Res Clin Rheumatol. 2016;30: 851-69.

9. Bai R, Zhang Y, Liu W, Ma C, Chen X, Yang J, et al. The relationship of ankylosing spondylitis and subclinical atherosclerosis: a systemic review and meta-analysis. Angiology. 2019;70:492-500.

10. Dagfinrud H, Kjeken I, Mowinckel P, Hagen KB, Kvien TK. Impact of functional impairment in ankylosing spondylitis: impairment, activity limitation, and participation restrictions. J Rheumatol. 2005;32:516-23.

11. Papagoras C, Voulgari P, Drosos A. Cardiovascular disease in the spondyloarthritides. Curr Vasc Pharmacol. 2020;18(5):473-87.

12. Papagoras C, Voulgari PV, Drosos AA. Atherosclerosis and cardiovascular disease in the spondyloarthritides, particularly ankylosing spondylitis and psoriatic arthritis. Clin Exp Rheumatol. 2013;31:612-20.

13. Bergfeldt L. HLA-B27-associated cardiac disease. Ann Intern Med [Internet]. 1997;127:621.

14. Peters MJL, Symmons DPM, McCarey D, Dijkmans BAC, Nicola P, Kvien TK, et al. EULAR evidencebased recommendations for cardiovascular risk management in patients with rheumatoid arthritis and other forms of inflammatory arthritis. Ann Rheum Dis. 2010;69:325-31.

15. Pletcher MJ, Moran AE. Cardiovascular risk assessment. Med Clin N Am. 2017;101:673-88.

16. Arts EEA, Popa CD, Den Broeder AA, Donders R, Sandoo A, Toms T, et al. Prediction of cardiovascular risk in rheumatoid arthritis: Performance of original and adapted SCORE algorithms. Ann Rheum Dis. 2016;75:674-80.

17. Crowson CS, Matteson EL, Roger VL, Therneau TM, Gabriel SE. Usefulness of risk scores to estimate the risk of cardiovascular disease in patients with rheumatoid arthritis. Am J Cardiol. 2012;110: 420-4.

18. Agca R, Heslinga SC, Rollefstad S, Heslinga M, McInnes IB, Peters MJL, et al. EULAR recommendations for cardiovascular disease risk management in patients with rheumatoid arthritis and other forms of inflammatory joint disorders: 2015/2016 update. Ann Rheum Dis. 2016;76:17-28.
19. Johnson KW, Torres Soto J, Glicksberg BS, Shameer $\mathrm{K}$, Miotto R, Ali M, et al. Artificial intelligence in cardiology. J Am Coll Cardiol. 2018;71:2668-79.

20. Narain R, Saxena S, Goyal AK. Cardiovascular risk prediction: a comparative study of framingham and quantum neural network based approach. Patient Prefer Adherence. 2016;10:1259-70.

21. D'Agostino RB, Vasan RS, Pencina MJ, Wolf PA, Cobain M, Massaro JM, et al. General cardiovascular risk profile for use in primary care: the Framingham heart study. Circulation. 2008;117:743-53.

22. Hippisley-Cox J, Coupland C, Vinogradova Y, Robson J, Minhas R, Sheikh A, et al. Predicting cardiovascular risk in England and Wales: prospective derivation and validation of QRISK2. BMJ. 2008;336:1475-82.

23. Hippisley-Cox J, Coupland C, Brindle P. Development and validation of QRISK3 risk prediction algorithms to estimate future risk of cardiovascular disease: prospective cohort study. BMJ. 2017;357: j2099.

24. Doukaki S, Caputo V, Bongiorno MR. Psoriasis and cardiovascular risk: assessment by cuore project risk score in Italian patients. Dermatol Res Pract. 2013;2013:389031.

25. Ridker PM, Buring JE, Rifai N, Cook NR. Development and validation of improved algorithms for the assessment of global cardiovascular risk in women: the Reynolds Risk Score. J Am Med Assoc. 2007;297: 611-9.

26. Woodward M, Brindle P, Tunsfall-Pedoe H. Adding social deprivation and family history to cardiovascular risk assessment: the ASSIGN score from the Scottish Heart Health Extended Cohort (SHHEC). Heart. 2007;93:172-6.

27. Perk J, Backer G De, Gohlke H, Graham I, J ZR-EH, Undefined. European Association for Cardiovascular Prevention \& Rehabilitation (EACPR); ESC committee for practice guidelines (CPG). European Guidelines on. 2012.

28. Batten A, Thorpe J, Piegari R, Rosland A-M. A resampling based grid search method to improve reliability and robustness of mixture-item response theory models of multimorbid high-risk patients. IEEE J Biomed Heal Inform [Internet] United States. 2019. https://doi.org/10.1109/JBHI.2019.2948734.

29. Chen DR, Kuo WJ, Chang RF, Moon WK, Lee CC. Use of the bootstrap technique with small training sets for computer-aided diagnosis in breast ultrasound. Ultrasound Med Biol. 2002;28:897-902. 
30. Didona D, Romano P. On bootstrapping machine learning performance predictors via analytical models. 2014. https://arxiv.org/abs/1410.5102. Cited 9 Jan 2020.

31. Henderson AR. The bootstrap: a technique for datadriven statistics. Using computer-intensive analyses to explore experimental data. Clin Chim Acta. 2005;359:1-26.

32. Liew JW, Ramiro S, Gensler LS. Cardiovascular morbidity and mortality in ankylosing spondylitis and psoriatic arthritis. Best Pract Res Clin Rheumatol. 2018;32:369-89.

33. Brophy S, Cooksey R, Atkinson M, Zhou SM, Husain MJ, Macey $S$, et al. No increased rate of acute myocardial infarction or stroke among patients with ankylosing spondylitis-a retrospective cohort study using routine data. Semin Arthritis Rheum [Internet]. 2012;42:140-5.

34. Eriksson JK, Jacobsson L, Bengtsson K, Askling J. Is ankylosing spondylitis a risk factor for cardiovascular disease, and how do these risks compare with those in rheumatoid arthritis? Ann Rheum Dis. 2017;76:364-70.

35. Bengtsson K, Forsblad-d'Elia H, Lie E, Klingberg E, Dehlin M, Exarchou S, et al. Are ankylosing spondylitis, psoriatic arthritis and undifferentiated spondyloarthritis associated with an increased risk of cardiovascular events? A prospective nationwide population-based cohort study. Arthritis Res Ther [Internet]. 2017;19. https://pubmed.ncbi.nlm.nih. gov/28521824/. Cited 24 Aug 2020.

36. Huang Y-P, Wang Y-H, Pan S-L. Increased risk of ischemic heart disease in young patients with newly diagnosed ankylosing spondylitis-a population-based longitudinal follow-up study. PLoS One [Internet]. 2013;8:e64155. https://doi.org/10. 1371/journal.pone.0064155.

37. Peters MJL, Visman I, Nielen MMJ, Van Dillen N, Verheij RA, Van Der Horst-Bruinsma IE, et al. Ankylosing spondylitis: a risk factor for myocardial infarction? Ann Rheum Dis [Internet]. 2010;69: 579-81.

38. Kim J, Kang U, Lee Y. Statistics and deep belief network-based cardiovascular risk prediction. Healthc Inform Res. 2017;23:169-75.

39. Benhamou M, Gossec L, Dougados M. Clinical relevance of C-reactive protein in ankylosing spondylitis and evaluation of the NSAIDs/coxibs' treatment effect on C-reactive protein. Rheumatology. 2009;49:536-41. 